\title{
Internal energy density of the critical three-state Potts model on the kagome lattice
}

\author{
Seung Ki Baek ${ }^{1, *}$ and Harri Mäkelä² \\ ${ }^{1}$ School of Physics, Korea Institute for Advanced Study, Seoul 130-722, Korea \\ ${ }^{2}$ QCD Labs, COMP Centre of Excellence, \\ Department of Applied Physics, Aalto University, \\ P.O. Box 13500, FI-00076 AALTO, Finland
}

\begin{abstract}
It has been conjectured that the internal energy density of the Potts model on a semi-infinite strip with a width $L$ does not have any finite-size corrections at the critical point $K=K_{c}$. By factorizing the transfer matrix for the kagome lattice with larger widths, we have found that this conjecture is not correct in that the internal energy density slightly varies with $L$ at the critical point. From this size dependence of the internal energy density, we obtain an upper bound as $K_{c}<1.0565615$, which is close to a recent estimate $K_{c}^{\mathrm{JS}}=1.0565600(7)$ by Jacobsen and Scullard arXiv:1204.0622. We also obtain a lower bound as $K_{c}>1.0560$ by calculating the correlation length along the strips.
\end{abstract}

PACS numbers: 64.60. De,05.70.Jk

\footnotetext{
* Corresponding author, E-mail: seungki@kias.re.kr
} 


\section{INTRODUCTION}

The $q$-state Potts model has served as a paradigmatic model in classical statistical physics since it allows to obtain exact and nontrivial analytic results. The model is defined by the following Hamiltonian,

$$
H=-J \sum_{\langle i j\rangle} \delta\left(S_{i}, S_{j}\right)
$$

where $J$ is an interaction constant, the summation runs over the nearest neighbors, $\delta$ is the Kronecker delta function, and $\operatorname{spin} S_{i}$ at site $i$ can take a value $0, \cdots, q-1$. For $q \leq 4$, the Potts model on a two-dimensional (2D) lattice undergoes a continuous phase transition at a certain inverse temperature $\beta=\beta_{c}(q)$. It is not a universal value but depends on the underlying lattice structure. One might say that locating $\beta_{c}$ is therefore of somewhat less importance than understanding universal behavior independent of lattice structures, but it can be understood as a question of decisive factors characterizing the critical point when the lattice symmetry is not high enough, which is still under debate. In a classical review article [1], the critical point of the Potts model on the kagome lattice is actually listed as the second unsolved problem about the Potts model. Wu's conjecture [1] suggests that by solving the following polynomial,

$$
v^{6}+6 v^{5}+9 v^{4}-2 q v^{3}-12 q v^{2}-6 q^{2} v-q^{3}=0
$$

where $v \equiv e^{K}-1$ with coupling strength $K \equiv \beta J$, one can get the critical point $K_{c} \equiv \beta_{c} J$. For $q=3$, the prediction is $K_{c} \approx 1.056494$, for example. Equation (2) is undoubtedly a good prediction, but slightly differs from Monte Carlo calculations [2] and series expansion results [3]. In a recent article [4], this problem was tackled by using an idea that certain spin models on an infinite strip with a finite width $L$ do not show finite-size corrections in the internal energy density $u_{L}$ [5]. By solving transfer matrices for two smallest widths $L=1$ and 2 [see Fig. 1(a) and 1(b)] and comparing the resulting internal energy densities, we conjectured $K_{c}^{\text {conj }}(q=3)=1.0565094269290 \ldots$ and $K_{c}^{\text {conj }}(q=4)=1.1493605872292 \ldots$ using finite-size scaling arguments. It was also suggested that one could directly check these conjectured values by calculating the internal energy density for a strip with $L=3$ shown in Fig. 1(c): If the calculated value for $q=3$ deviates from $u_{L=3}^{\text {conj }}(q=3)=-1.6295437063996$ given in Ref. [4], our determination based on the assumption of $L$-independent $u_{L}\left(K_{c}\right)$ must be incorrect. In this work, we carry out this calculation by using the factorization 
(a)

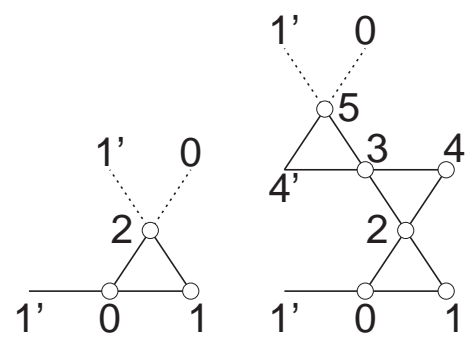

(b)
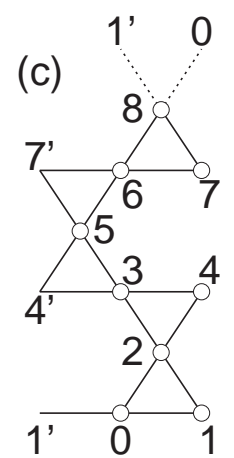

FIG. 1. Unit cells of the kagome lattice with different widths (a) $L=1$, (b) $L=2$, and (c) $L=3$. The dotted lines mean the periodic boundary condition so that $1^{\prime}$ and 0 on the top are connected to $1^{\prime}$ and 0 at the bottom, respectively.

technique [6] and report that $u_{L}$ at $K_{c}^{\operatorname{conj}}(q=3)$ is different from the conjectured value $u_{L}^{\text {conj }}(q=3)$ given above. It means that the absence of finite-size corrections assumed in Ref. [4] is not true in general except for $q=2$. This was recently pointed out by Jacobsen

and Scullard [7]: They mentioned the existence of finite-size corrections for $q \neq 2$, and our conjectured values are not compatible with their results obtained by improving the polynomial in Eq. (2) systematically with larger subgraphs of the kagome lattice. So the conjecture in Ref. [4] is disproved, which is the main result of this work. The second is that it is nevertheless possible to find bounds for $K_{c}$ by using the size dependence of numerical results. Our upper bound in this work is $K_{c}<1.0565615$, and a lower bound is obtained as $K_{c}>1.0560$, which are consistent with the value $K_{c}^{\mathrm{JS}}=1.0565600(7)$ estimated by Jacobsen and Scullard [7].

\section{METHOD AND RESULT}

It is straightforward in principle to construct a transfer matrix $T$ describing the threestate Potts model on an infinite strip with a width $L$. The problem is that it gets too large as $L$ grows. Therefore, we need to factorize $T$ into sparse matrices [6] and then use the power iteration method to get the largest eigenvalue and the corresponding left and right eigenvectors. The factorization means that a transfer matrix of a unit cell of width $L$ as 
TABLE I. One possible correct order of adding spins for $L=3$ [Fig. 11(c)]. A link between spins $i$ and $j$ is denoted by $(i, j)$.

\begin{tabular}{cc}
\hline spins added & links added \\
\hline 0 & $\left(0,1^{\prime}\right)$ \\
2 & $(2,0)$ \\
3 & $\left(3,4^{\prime}\right),(3,2)$ \\
5 & $\left(5,4^{\prime}\right),\left(5,7^{\prime}\right),(5,3)$ \\
4 & $(4,3),(4,2)$ \\
6 & $\left(6,7^{\prime}\right),(6,5)$ \\
7 & $(7,6)$ \\
8 & $\left(8,1^{\prime}\right),(8,0),(8,6),(8,7)$ \\
1 & $(1,0),(1,2)$ \\
\hline
\end{tabular}

in Fig. 1 is equivalent to a product of $3 L$ sparse matrices each of which describes adding a single spin. The advantage of the factorization lies in the fact that multiplying a vector by the sparse matrices one after another is much faster than dealing with the original dense matrix at once, because only a few elements of the vector are affected by a sparse matrix. Note that we have to be careful about the order of adding spins under the periodic boundary condition along the vertical direction, because each spin addition amounts to replacing a spin value in a layer by a new one. For example, in Fig. 1(a), adding spin 1 replaces the old value of spin $1^{\prime}$, eliminating its information. For that reason, one possible correct order for $L=1$ is $0 \rightarrow 2 \rightarrow 1$, where spin 1 is added after spin 2 , because spin 2 should have a link to spin $1^{\prime}$ before spin 1 replaces it, according to the periodic boundary condition [see the dotted line between spin 2 and spin $1^{\prime}$ in Fig. 1(a)]. For the same reason, one correct order for $L=2$ is $0 \rightarrow 2 \rightarrow 3 \rightarrow 5 \rightarrow 4 \rightarrow 1$, and Table 1 shows a possible order for $L=3$ in detail. This approach can be extended to larger $L$ 's in a straightforward manner. For direct comparison, we have chosen the same transfer direction as in Ref. [4]. It is notable that Ref. [7] also carried out transfer matrix calculations to assess their approximation but in a different transfer direction.

As we multiply an arbitrary vector by the transfer matrix $T$ over and over again, it almost surely converges to an eigenvector with the largest eigenvalue $\lambda_{1}$ of $T$. Once we 

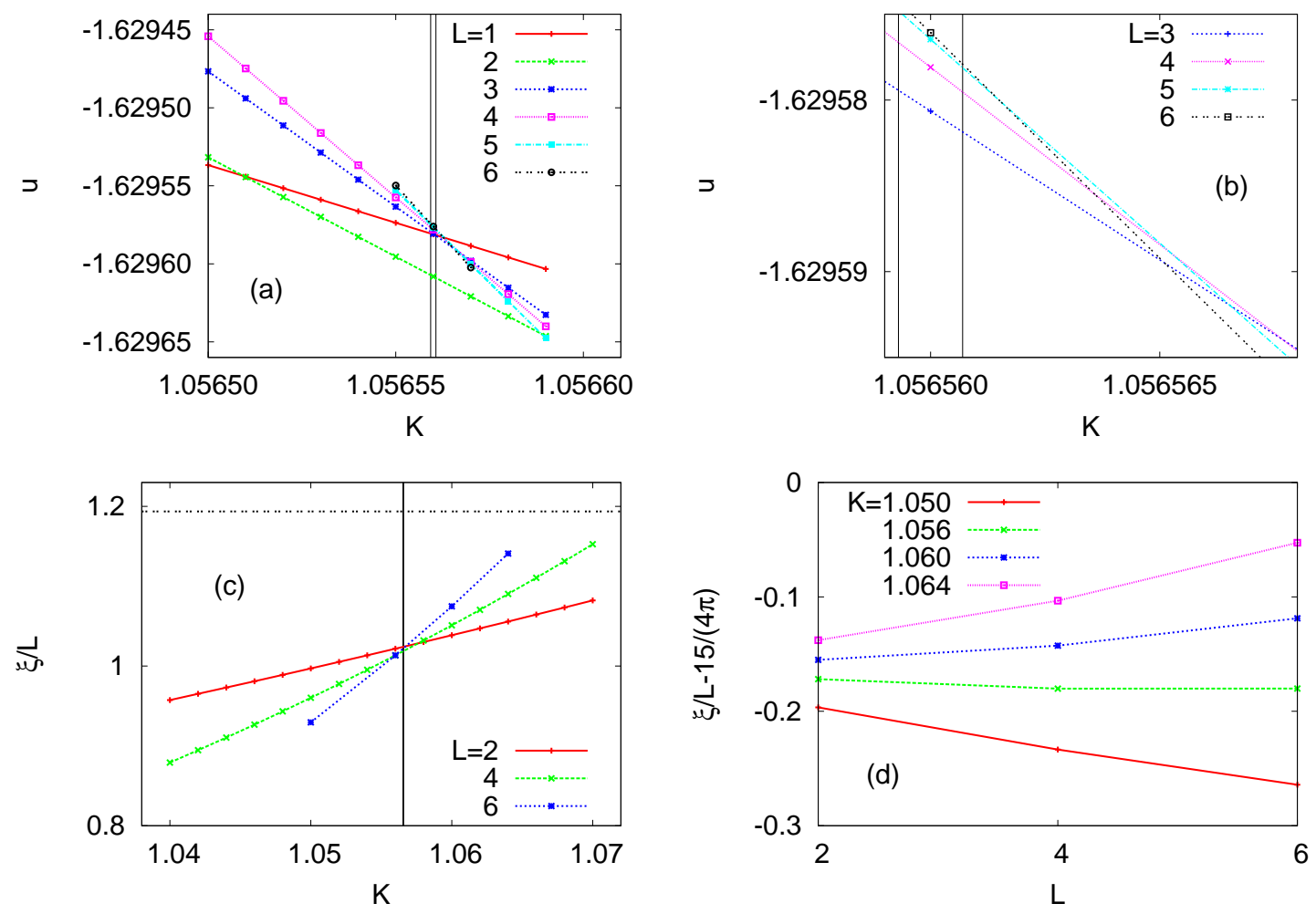

FIG. 2. (Color online) (a) Internal energy density as a function of $K$ for each strip width $L$ and (b) its zoomed view. (c) Correlation length $\xi$ divided by $L$, where the dotted horizontal line means $1 /(\pi \eta)=15 /(4 \pi)$. The vertical lines in panels (a) to (c) indicate the range of $K_{c}$ estimated by Jacobsen and Scullard [7]. (d) As $L$ increases, the convergence of $\xi / L$ to $15 /(4 \pi)$ is observed for $K>1.056$.

have obtained the left eigenvector $\mathbf{v}_{L}$ and the right eigenvector $\mathbf{v}_{R}$ corresponding to $\lambda_{1}$, we are able to get $u_{L}=-\left(3 L \lambda_{1}\right)^{-1} \partial \lambda_{1} / \partial \beta=-\left(3 L \lambda_{1}\right)^{-1} \mathbf{v}_{L}^{*} \cdot(\partial T / \partial \beta) \cdot \mathbf{v}_{R}$, where $*$ means complex conjugate transpose. The factor of $3 L$ appears since it is the number of spins for constructing $L$ layers as depicted in Fig. 1, The numerical error $\delta u_{L}$ in the internal energy density increases with $L$ and it is estimated as $\delta u_{L} \sim O\left(10^{-11}\right)$ for $L=5$ and $O\left(10^{-9}\right)$ for $L=6$. For $L=3$, the internal energy density at $K_{c}^{\text {conj }}(q=3)$ obtained by this method reads $u_{L=3}^{\text {power }}=-1.62949 \ldots$, which clearly deviates from $u_{L=3}^{\text {conj }}=-1.62954 \ldots$ conjectured in Ref. [4], disproving the conjecture on the lack of finite-size corrections in $u_{L}\left(K_{c}\right)$.

Our results up to $L=6$ are depicted in Fig. 2(a). The line of $L=1$ behaves differently from the others since the size is too small and should therefore be discarded from 
consideration. The crossing points of lines for $L \geq 2$ gradually converge to $K_{c}$ from above [Fig. 2(b)]. It is therefore plausible that $K_{c}$ is located to the left of the crossing between $L=5$ and $L=6$ at $K \approx 1.0565615$. Although the size-dependence of $u_{L}$ is known for large $L$ at the critical point, our fitting result to estimate $K_{c}$ from this critical scaling behavior is inconclusive because sub-leading corrections are not negligible for such small $L$ 's.

In order to estimate a lower bound, we use correlation length $\xi=\left[\ln \left(\lambda_{1} / \lambda_{2}\right)\right]^{-1}$, where $\lambda_{2}$ is the second largest eigenvalue of $T$. The second largest eigenvalue can be found by applying the power iteration method to a vector orthogonal to $\mathbf{v}_{R}$, the eigenvector associated with $\lambda_{1}$. The numerical error in $\xi$ is estimated as $O\left(10^{-11}\right)$ for $L=5$ and $O\left(10^{-7}\right)$ for $L=6$. As $L$ increases, it is known that $\xi / L$ converges to $1 /(\pi \eta)=15 /(4 \pi) \approx 1.194$ at criticality, where $\eta$ is the critical exponent for the two-spin correlation function [8]. Since $\xi$ is an increasing function of $K$ and the lines cross each other below 1/( $\pi \eta)$ [see Fig. 2(c)], the crossing point will approach $K_{c}$ from below if $L$ is large enough. We thereby infer a lower bound as $K_{c}>1.0560$, where the lines of $L=4$ and $L=6$ cross each other. The convergence of $\xi / L$ to $15 /(4 \pi)$ also suggests that the borderline is around $K=1.056$ [Fig. 2(d)]. Note that we compare results from even $L$ 's here because the behavior of $\lambda_{2}$ seems more sensitive to the boundary condition in the vertical direction than that of $\lambda_{1}$.

\section{SUMMARY}

In summary, we have numerically calculated internal energy densities near $K=K_{c}^{\text {conj }}$ for the three-state $(q=3)$ Potts model on the kagome-type strips with various widths. The result disproves our previous conjecture that the internal energy density has no finite-size corrections at the critical point. The size dependence suggests $1.0560<K_{c}<1.0565615$, which is consistent with the recent estimate $K_{c}^{\mathrm{JS}}=1.0565600(7)$ [7].

\section{ACKNOWLEDGMENTS}

H.M. was supported by the Alfred Kordelin Foundation and the Academy of Finland through its Centres of Excellence Program (Project No. 251748). We thank KIAS Center 
for Advanced Computation for providing computing resources.

[1] F. Y. Wu, Rev. Mod. Phys. 54, 235 (1982).

[2] R. M. Ziff and P. N. Suding, J. Phys. A 30, 5351 (1997).

[3] I. Jensen, A. J. Guttmann, and I. G. Enting, J. Phys. A 30, 8067 (1997).

[4] S. K. Baek, H. Mäkelä, P. Minnhagen, and B. J. Kim, Phys. Rev. E 83, 061104 (2011).

[5] J. Wosiek, Phys. Rev. B 49, 15023 (1994).

[6] H. W. J. Blöte and M. P. Nightingale, Physica A 112, 405 (1982); H. W. J. Blöte and B. Nienhuis, J. Phys. A 22, 1415 (1989); D. P. Foster, C. Gérard, and I. Puha, ibid. 34, 5183 (2001).

[7] J. L. Jacobsen and C. R. Scullard, J. Phys. A 45, 494003 (2012).

[8] J. Cardy, J. Phys. A 17, L385 (1984). 